\title{
Thermally and field-driven spin-state transitions in $\left(\mathrm{Pr}_{1-y} \mathrm{Y}_{y}\right)_{0.7} \mathrm{Ca}_{0.3} \mathrm{CoO}_{3}$
}

\author{
M. Maryško, ${ }^{1, a)}$ Z. Jirák, ${ }^{1}$ K. Knížek, ${ }^{1}$ P. Novák, ${ }^{1}$ J. Hejtmánek, ${ }^{1}$ T. Naito, ${ }^{2}$ H. Sasaki, ${ }^{2}$ \\ and H. Fujishiro ${ }^{2}$ \\ ${ }^{1}$ Institute of Physics, ACSR, Na Slovance 2, 18221 Praha 8, Czech Republic \\ ${ }^{2}$ Faculty of Engineering, Iwane University, 4-3-5 Ueda, Morioka 020-8551, Japan
}

(Presented 17 November 2010; received 12 October 2010; accepted 27 November 2010; published online 1 April 2011)

\begin{abstract}
The first-order character of the transition from the low-to-intermediate spin state in the system $\left(\operatorname{Pr}_{1-y} \mathrm{Y}_{y}\right)_{0.7} \mathrm{Ca}_{0.3} \mathrm{CoO}_{3}(y=0.075-0.15)$ has been verified by magnetization measurements. For $y=0.075$, the transition manifests itself in $\chi(T)$ steps at temperatures depending on external fields. In this case, isothermal measurements show a field-driven transition exhibiting a field hysteresis. The entropy contribution is evaluated and discussed. Qualitative analysis based on a two-level model suggests that the observed influence of the magnetic field can be explained only by assuming the presence of exchange interactions. (c) 2011 American Institute of Physics. [doi:10.1063/1.3559485]
\end{abstract}

\section{INTRODUCTION}

The spin-state transitions (SST) in $\mathrm{LaCoO}_{3}$-type cobaltites concern the $\mathrm{Co}^{3+}$ ions, which may occur in the low-spin (LS) $s=0$, intermediate (IS) $s=1$, or high-spin $s=2$ states. A specific case of simultaneous first-order SST and metalinsulator transition, accompanied by a large volume change, was reported on the mixed valency cobaltite $\operatorname{Pr}_{0.5} \mathrm{Ca}_{0.5} \mathrm{CoO}_{3}$ (Ref. 1) and related systems of lower calcium doping with $\mathrm{Pr}$ substituted by smaller rare earths (see, e.g., Ref. 2). Recently, the exclusive role of praseodymium for such a type of transition has been elucidated. ${ }^{3-5}$ Based on the electronic structure calculations and detailed analysis of the structural, electrical, magnetic, and specific heat data, it has been found that the transition is accompanied by a significant charge transfer between cobalt and praseodymium ions. In particular for the system $\left(\operatorname{Pr}_{1-y} \mathrm{Y}_{y}\right)_{0.7} \mathrm{Ca}_{03} \mathrm{CoO}_{3}$, about onethird of the $\mathrm{Pr}^{3+}$ ions changes their valency to $\mathrm{Pe}^{4+}$ during the SST on decreasing temperature. ${ }^{4}$ In the present contribution we follow previous works ${ }^{2,4}$ and bring a detailed study of the first-order SST and its dependence on the applied magnetic field, taking a special emphasis on the composition $y=0.075$. The new result is the observation of a fielddriven SST, which can be explained by the presence of the exchange interaction acting on the paramagnetic IS $\mathrm{Co}^{3+}$ ion.

\section{EXPERIMENT}

In our experiments we used the ceramic samples of $\left(\operatorname{Pr}_{1-y} \mathrm{Y}_{y}\right)_{0.7} \mathrm{Ca}_{0.3} \mathrm{CoO}_{3}(y=0.075,0.1,0.15)$ described previously in Ref. 4. The magnetic measurements were carried out by means of the superconducting quantum interference device magnetometers MPMS XL and MPMS-5S. The temperature dependence of the susceptibility $\chi$ under an applied field $1 \mathrm{kOe}$ was measured between 5 and $300 \mathrm{~K}$ using a temperature sweep rate of $2 \mathrm{~K} / \mathrm{min}$. In increasing temperature

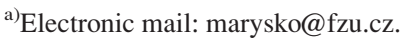

the SST manifests itself in an increase of the susceptibility by $\Delta \chi=\Delta m / H$. In Fig. 1 (a) the results are shown between 20 and $200 \mathrm{~K}$, where we observe the SST at the temperatures $T_{s} \approx 64,93$, and $132 \mathrm{~K}$ with $\Delta \chi \approx 0.020,0.0093$, and 0.0058 emu mol ${ }^{-1} \mathrm{Oe}^{-1}$ for $y=0.075,0.1$, and 0.15 , respectively. At the same temperatures a pronounced peak of the specific heat has been observed, very sharp for the former two samples and more diffuse for the latter one (see Ref. 4).

As a second step, we carried out the measurements around $T_{s}$ employing smaller sweep rates that enable the precise determination of thermal hysteresis. For the $y=0.075$ sample, where the SST is very sharp, we found that the reliable results, which are not dependent on the sweep rate, can be achieved with the rate $0.007 \mathrm{~K} / \mathrm{min}$. An example of thermal hysteresis curve (THC) is given in Fig. 1(b), which shows the $\chi(T)$ plot measured at $H=10 \mathrm{kOe}$ during warming and subsequent cooling. At the temperatures $T_{\mathrm{su}}$ and $T_{\mathrm{sd}}$ we see an almost step increase and decrease of the susceptibility with the thermal hysteresis $\Delta T=T_{\mathrm{su}}-T_{\mathrm{sd}}$ and the average SST temperature $T_{s}=0.5\left(T_{\mathrm{su}}+T_{\mathrm{sd}}\right)$. Here, we obtained $T_{s}=64.04$ and $\Delta T=0.35 \mathrm{~K}$. The same type of experiment was then performed for several static magnetic fields, including $H=0$, where the THC was evaluated from the real part of the ac susceptibility measured with a driving ac field 3.9 Oe (the imaginary part was too small to be detected). In accordance with expectation, $T_{s}$ decreases with increasing field $H$ and the dependence can be well approximated by the relation $T_{s}(H)=T_{s}(0)-A H^{2}$ [inset of Fig. 1(b)]. The value of $\Delta T$ increases with increasing field $H$ from 0.19 Oe $(H=0)$ to $0.48(H=70 \mathrm{kOe})$. The data on $T_{s}(0), A$, and the average value of $\Delta T$ are listed in Table I.

For $y=0.1$ and 0.15 the character of the SST becomes more continuous as can be seen in Fig. 1(a). Analogously as in the case of $y=0.075$, we performed the measurements of the THC around $T_{s}$ using the sweep rates of $0.15 \mathrm{~K} / \mathrm{min}$ $(y=0.1)$ and $0.2 \mathrm{~K} / \mathrm{min}(y=0.15)$. In both cases, $\Delta T$ and $A$ are very small (Table I). The results for $y=0.15$ are exemplified in Fig. 1(c). 

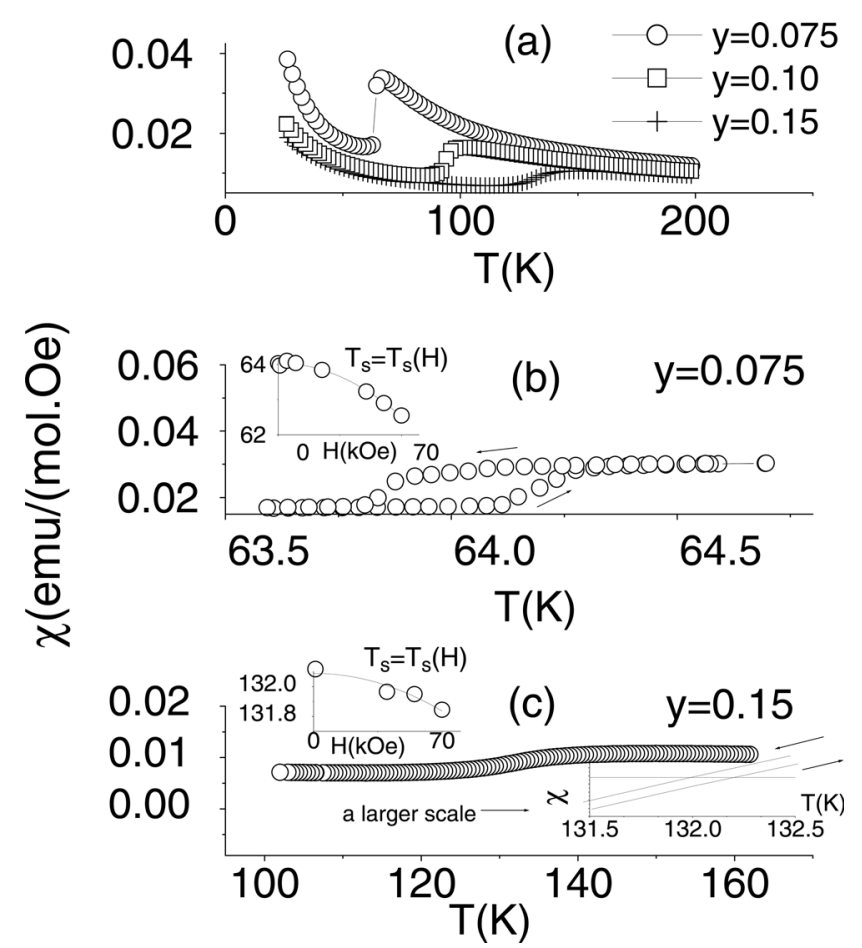

FIG. 1. (a) Temperature dependence of the molar susceptibility measured under an applied field $1 \mathrm{kOe}$; (b) thermal hysteresis for $y=0.075$ at $H=10$ $\mathrm{kOe}$, in the inset the dependence of average $T_{S}$ on magnetic field $H$; (c) the same for $y=0.15$ and, in the right inset, the part of the thermal hysteresis curve displayed on a larger scale.

Now we focus our attention on the isothermal $m(H)$ dependencies taken at temperatures near $T_{s}(0)$. In contrast to the $y=0.1$ and 0.15 samples, we find that for $y=0.075$ the hysteresis curves indicating field-driven SST transitions. At $T=64 \mathrm{~K}$ the dependence $m(H)$ is linear, corresponding to the field-independent susceptibility $m / H$ of paramagnetic ions $\mathrm{Co}^{3+}, \mathrm{Co}^{4+}$, and $\mathrm{Pr}^{3+}$ [Fig. 2(a)]. With decreasing temperature, a metamagnetic-like transition becomes evident and, due to hysteresis, the $m(H)$ dependence transforms into a closed loop. The contribution of the SST, obtained by subtracting linear dependence corresponding to the paramagnetic background, is illustrated by a dotted line in Fig. 2(b). At $T=63.3 \mathrm{~K}$, the average field hysteresis makes $\Delta H \approx 13$ $\mathrm{kOe}$.

\section{DISCUSSION}

The finite values of $\Delta T$ and $\Delta H$ seen in Figs. 1(b) and 2(b) indicate the first-order character of SST. In this case, when increasing temperature a latent heat $\Delta q$ must be transferred to a system and its entropy increases by $\Delta S=\Delta q / T$. The entropy change $\Delta S$ can be determined using the Clausius-Clapeyron equation $d T_{s} / d H=T \Delta m / \Delta q$. After

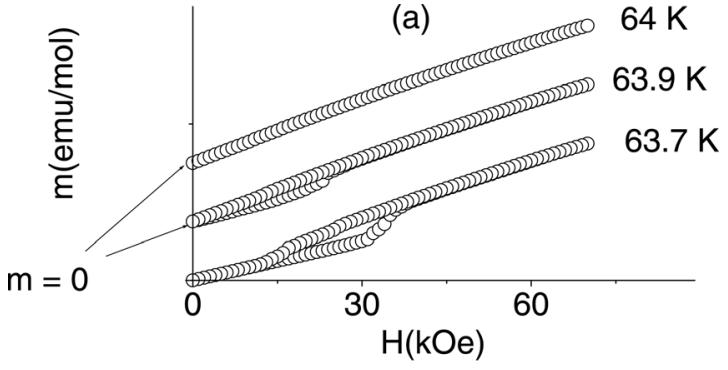

(b)

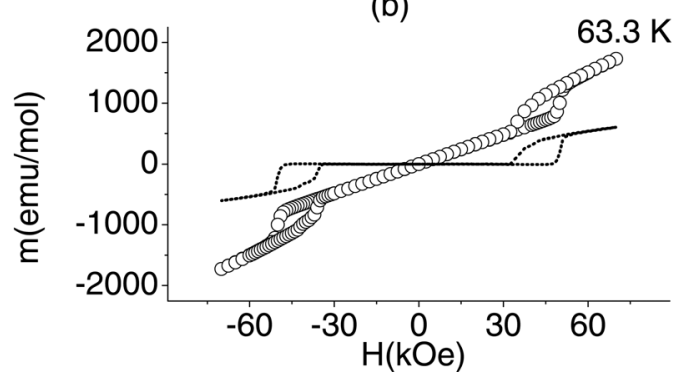

FIG. 2. The hysteresis loops for $y=0.075$ measured at several temperatures near $T_{s}(0)$; the hysteresis loop corrected for a paramagnetic background is shown by the dotted curve.

setting $\Delta m=H \Delta \chi$ and integrating, the Clausius-Clapeyron equation transforms to $T_{s}=T_{s}(0)-A H^{2}$, where $A=\Delta \chi /(2 \Delta S)$ and $T_{s}(0)$ is the SST temperature for $H=0$. The determined values of $\Delta S$ are included in Table I.

The important experimental fact is a step-like increase of $\chi$ in increasing both temperature and field. To elucidate this finding, we restrict our study to the $\mathrm{Co}^{3+}$ ion and its transition from the LS to IS state. We start from a thermodynamic two-level model of SST. ${ }^{6-8}$ Using the notation in Ref. 7 the system can be characterized by two energy parameters $E$ and $\varepsilon$, where $E$ is the distance between the lowest LS singlet level and excited IS level with triple orbital and triple spin degeneracy. The parameter $\varepsilon$ represents an energy gain due a change of the interatomic distances. The model calculation yields population $n$ of the IS $\mathrm{Co}^{3+}$ ions, which should be proportional to $\Delta \chi$. The essential point is that the excitation is accelerated compared to simple Boltzmann statistics (the LS-IS gap shrinks gradually) and becomes even of firstorder type, because the lattice volume adjusts to increasing number $n$. For $y=0.075$ the calculation with spin $s=1$, $g=2,2 l+1=3, r=\varepsilon / E=0.51$, and $E$ (Table I) yields the function $n(T)$ exhibiting a step at $T=64 \mathrm{~K}$, but its dependence on $H$ field is very small $(0.16 \mathrm{~K}$ for $\delta H=70 \mathrm{kOe})$. A reasonable agreement with experiment can be achieved only if we take into account a positive molecular field (ferromagnetic exchange interaction) acting on the paramagnetic IS $\mathrm{Co}^{3+}$ ion. This interaction can be approximately included

TABLE I. Parameters $T_{s}(0), A, \Delta \chi, \Delta S$, and $\Delta T$ deduced from experiment and $E / k B$ used in the model calculation.

\begin{tabular}{|c|c|c|c|c|c|c|}
\hline$y$ & $T_{s}(0)(\mathrm{K})$ & $A \times 10^{4}\left(\mathrm{~K} \mathrm{kOe}^{-2}\right)$ & $\Delta \chi\left(\mathrm{emu} \mathrm{mol}{ }^{-1} \mathrm{Oe}^{-1}\right)$ & $\Delta S\left(\mathrm{~J} \mathrm{~mol}^{-1} \mathrm{~K}^{-1} 1\right)$ & $\Delta T(\mathrm{~K})$ & $E / k B(\mathrm{~K})$ \\
\hline 0.075 & 64.04 & 3.10 & 0.02 & 3.23 & 0.35 & 280.3 \\
\hline 0.1 & 93.13 & 1.43 & 0.0093 & 3.25 & 0.35 & 365.4 \\
\hline 0.15 & 132.32 & 0.51 & 0.0058 & 5.68 & 0.23 & 538.4 \\
\hline
\end{tabular}



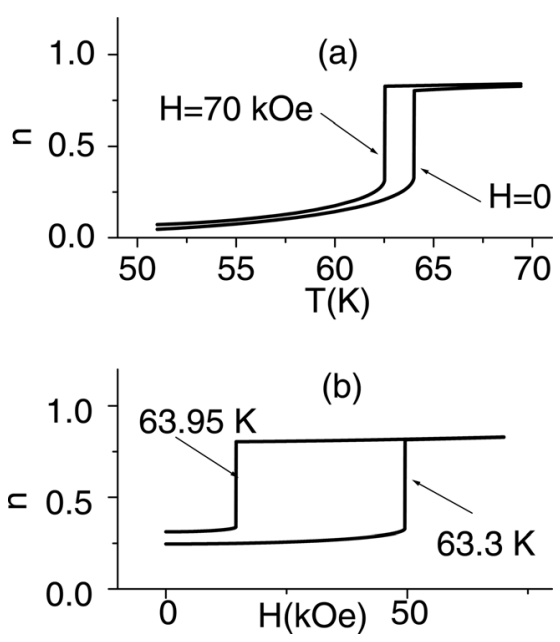

FIG. 3. Results of the calculations according to the two-level model: the temperature (a) and field (b) dependence of the occupation number $n$.

by changing the factor $\mu_{B} H / k T$ [see expression (35) in Ref. 7] by replacing $T$ by $T-\vartheta$, where $\vartheta$ is a temperature characterizing the interaction. Using $\vartheta=42 \mathrm{~K}$ we obtain the functions $n(T)$, for which $T_{s}(0)=64 \quad \mathrm{~K}$ and $T_{s}(70 \mathrm{kOe})=62.5 \mathrm{~K}$ [Fig. 3(a)]. In Fig. 3(b) we show that the calculated functions $n(H)$ for $T=63.3$ and $63.95 \mathrm{~K} \mathrm{ex}$ hibit step changes at 49.5 and $5.5 \mathrm{kOe}$, respectively. For $y=0.1$ and 0.15 the calculated $n(T)$ dependencies were in a relatively good agreement with experiment for parameters for $r=0.44$ and 0.46 and $\vartheta=51$ and $48.6 \mathrm{~K}$, respectively.

The final comment concerns the $\Delta S$ values presented in Table I. They are significantly lower than the two-level model of local excitations predicts for $\left(\operatorname{Pr}_{1-y} \mathrm{Y}_{y}\right)_{0.7} \mathrm{Ca}_{0.3}$ $\mathrm{CoO}_{3}-\Delta S=0.7 R \ln 9=12.78 \mathrm{~J} \mathrm{~mol}^{-1} \mathrm{~K}^{-1}$. This discrepancy can be understood considering the metallic character of phase above $T_{s}$. We note that the excited IS states do not exist as bare $\mathrm{Co}^{3+}$ species. They provide $e_{g}$ carriers that are delocalized over surrounding $\mathrm{Co}^{4+}$ sites, forming a droplet of the metallic phase of $t_{2 g}^{5} \sigma^{*}$ character. In that case the relevant entropy retains only the spin degeneracy part and instead of common configuration term, $S=R(0.7 \ln 0.7$ $+0.3 \ln 0.3$ ), it contains a term that derives from density of states of the final phase, $S=\gamma T$ with $\gamma$ of about $0.040 \mathrm{~J}$ $\mathrm{mol}^{-1} \mathrm{~K}^{-2}$ (see, e.g., Ref. 9). For $y=0.075$ with $T_{s} \approx 64 \mathrm{~K}$ one may, therefore, estimate $\Delta S=\gamma T+0.7 R \ln 3-R$ $(0.7 \ln 0.7+0.3 \ln 0.3)=2.56+6.40-5.08=3.88 \mathrm{~J} \mathrm{~mol}^{-1}$ $\mathrm{K}^{-1}$, in reasonable agreement with the experimental value of $3.23 \mathrm{~J} \mathrm{~mol}^{-1} \mathrm{~K}^{-1}$.

In summary, the spin-state transitions in $\left(\operatorname{Pr}_{1-y} \mathrm{Y}_{y}\right)_{0.7}$ $\mathrm{Ca}_{0.3} \mathrm{CoO}_{3}$ with $y=0.075-0.15$ exhibit a finite small hysteresis and the transition temperature depends on the magnetic field. The latter dependence enables the determination of the entropy contribution which is compared with the theoretical value. For $y=0.075$ a field-driven transition is observed for cobaltites. The simple two-level model modified for the presence of the exchange interaction is used to qualitatively describe a step-like dependencies of the susceptibility on temperature and magnetic field.

\section{ACKNOWLEDGMENTS}

The work was supported under Project No. 202/09/0421 of the Grant Agency of the Czech Republic and GA ASCR Grant No. 400720619.

${ }^{1}$ S. Tsubouchi, T. Kyômen, M. Itoh, P. Ganguly, M. Oguni, Y. Shimojo, Y. Morii, and Y. Ishii, Phys. Rev. B 66, 052418 (2002).

${ }^{2}$ T. Naito, H. Sasaki, and H. Fujishiro, J. Phys. Soc. Jpn. 79, 034710 (2010).

${ }^{3}$ K. Knížek, J. Hejtmánek, P. Novák, and Z. Jirák, Phys. Rev. B 81, 155113 (2010).

${ }^{4}$ J. Hejtmánek, E. Šantavá, K. Knížek, M. Maryško, Z. Jirák, T. Naito, H. Sasaki, and H. Fujishiro, Phys. Rev. B 82, 165107 (2010).

${ }^{5}$ A. J. Barón-González, C. Frontera, J. L. García-Muñoz, J. Blasco, and C. Ritter, Phys. Rev. B 81, 054427 (2010).

${ }^{6}$ R. A. Bari and J. Sivardière, Phys. Rev. B 5, 4466 (1972).

${ }^{7}$ S. W. Biernacki and B. Clerjaud, Phys. Rev. B 72, 024406 (2005).

${ }^{8}$ S. W. Biernacki, Phys. Rev. B 74, 184420 (2006).

${ }^{9}$ Z. Jirák, J. Hejtmánek, K. Knížek, and M. Veverka, Phys. Rev. B 78, 014432 (2008). 